\title{
Secular Trends in the Prevalence of Abdominal Obesity Among Chinese Adults With Normal Weight, 1993-2015
}

\author{
Xingxing Sun \\ Tongji Hospital, Tongji Medical College of Huazhong University of Science and Technology \\ Tingting Du ( $\square$ aduttsxx@163.com ) \\ Tongji Hospital, Tongji Medical College of Huazhong University of Science and Technology
}

\author{
Research Article \\ Keywords: Body mass index, waist circumference, abdominal obesity, general obesity \\ Posted Date: December 23rd, 2020 \\ DOI: https://doi.org/10.21203/rs.3.rs-127698/v1 \\ License: (c) (i) This work is licensed under a Creative Commons Attribution 4.0 International License. Read Full License
}

Version of Record: A version of this preprint was published at Scientific Reports on August 12th, 2021. See the published version at https://doi.org/10.1038/s41598-021-95777-y. 


\section{Abstract}

Background: We aimed to analyze updated prevalence data on abdominal obesity trends among Chinese adults with a normal body mass index (BMI).

Methods: We used data from the China Health and Nutrition Survey (CHNS) conducted between 1993 and 2015.

Results: Over the 23-year period, the age-standardized mean WC values showed a significant increasing trend among Chinese adults with BMI < 25 $\mathrm{kg} / \mathrm{m}^{2}$, with the mean value increased from $73.8 \mathrm{~cm}$ to $78.2 \mathrm{~cm}$ ( $P$ for trend $<0.001$ ). During the period of $1993-2015$, the age-standardized prevalence of abdominal obesity increased from $11.2 \%$ to $24.8 \%$ ( $P$ for trend $<0.001$ ). Significant increases were observed in both sexes, all age groups, rural and urban residents, and all educational attainment groups (all P for trends $<0.001$ ), with a greater relative increase noted among men, younger participants, and rural residents. Similar significant trends were noted when a more stringent BMl $<23 \mathrm{~kg} / \mathrm{m}^{2} \mathrm{cut}$ point (Asian cut point) was applied.

Conclusions: The mean WC and the prevalence of abdominal obesity among Chinese adults with normal BMI increased continuously from 1993 to 2015. The upward trends were noted in both sexes, all age groups, rural and urban regions, and all educational attainment groups.

\section{Introduction}

With the dramatic shift in diet from traditional to Western dietary patterns and a steep decline in physical activity levels that have followed the rapid urbanization and industrialization, increasing rates of obesity in China are especially alarming ${ }^{1,2}$. China has moved from 60 th place for men and 41st place for women in 1975 to second for both men and women in 2014 in the worldwide ranking of the number of severely obese individuals ${ }^{3}$. Approximately one in five obesity individuals worldwide are Chinese ${ }^{2}$. The fast increase in the prevalence of obesity in China has been accompanied by marked increases in an expanding set of chronic diseases, including diabetes mellitus, cardiovascular disease, chronic kidney disease, many cancers, and an array of cognitive and musculoskeletal disorders ${ }^{4}$.

Normally, body mass index (BMI) has been used as a proxy for obesity in the population. However, abdominal obesity assessed by waist circumference (WC) is more pathogenic and thus is more closely associated with type 2 diabetes, cardiovascular disease, and cancer mortality than general obesity ${ }^{5-8}$. Further, BMI is a poor indicator of body fat distribution, as evidenced by the occurrence of the variation in the burden of diabetes mellitus, and cardiovascular disease among individuals with similar $\mathrm{BMI}^{9-11}$. In addition, type 2 diabetes, and cardiovascular disease are not rare diseases in persons with normal BM ${ }^{12}$. For example, $7.6 \%$ of Chinese individuals with normal BMI suffered from diabetes ${ }^{9,12}$, more than $20 \%$ of the normal weight population encountered with metabolic disorders ${ }^{9}$, and globally, $39 \%$ of deaths and $37 \%$ of disability-adjusted life-years occurred among nonobese persons ${ }^{4}$. Taken together, it is important to know the trends in abdominal obesity. Although trends in abdominal obesity in China have been evaluated in several studies ${ }^{1,13}$, very few studies describe the trends in abdominal obesity among individuals with normal BMI ${ }^{14}$. Given that Chinese population, who, despite being generally less obese, are more prone to visceral fat accumulation compared with western populations ${ }^{15}$, the increase in WC was more pronounced than the increase in $\mathrm{BMI}$ at given periods in China ${ }^{13}$, and that a considerable disease burden existed in people with normal BMI, it is imperative to study the prevailing trends in abdominal obesity among Chinese people with normal BMI. Our previous study reported an upward trend from 1993 to 2009 in the prevalence of abdominal obesity among Chinese people with normal BMI irrespective of sex, age, rural/urban settings, and education levels ${ }^{14}$. To get a more comprehensive understanding of the trends in abdominal obesity among Chinese people with normal BMI over the years from 2009 to 2015, this study presents new data for recent trends in abdominal obesity from 2009 to 2015.

\section{Methods Study design}

We used data from the China Health and Nutrition Survey (CHNS) for our analysis. The CHNS is a series of cross-sectional household-based surveys conducted by the Carolina Population Center at the University of North Carolina at Chapel Hill and the National Institute of Nutrition and Food Safety at the Chinese Center for Disease Control and Prevention. Full details of the study have been described elsewhere ${ }^{1,14,16}$. Briefly, the CHNS rounds were conducted in 1989, 1991, 1993, 1997, 2000, 2004, 2006, 2009, 2011, 2015, and 2019. A stratified multistage, random cluster sampling was employed to draw study sample from 12 provinces (Liaoning, Heilongjiang, Shandong, Shaanxi, Henan, Hubei, Hunan, Guangxi, Guizhou, Jiangsu, Yunnan and, Zhejiang), that vary significantly in terms of geography, economic development, and health status. The survey procedures were reviewed and approved by the institutional review committees of the University of North Carolina at Chapel Hill, and the National Institute of Nutrition and Food Safety, Chinese Center for Disease Control and Prevention in accordance with the ethical standards laid down in the 1964 Declaration of Helsinki and its later amendments. Informed consent was obtained from all participants and/or their legal guardians.

\section{Study population}


All participants were asked to complete a structured questionnaire which provided information on age, sex, place of residence, educational attainment, and medical history. Participants were included in the present analysis if they were 18 years or older. Exclusion criteria included pregnancy, and with missing information on age, WC, BMI, or blood pressure (BP), and with extreme or implausible WC (51 cm or $190 \mathrm{~cm})$, BMI, or BP values. The remaining analytic sample sizes for the current study were 7745 in 1993, 8351 in 1997, 9331 in 2000,8993 in 2004,8856 in 2006 , 9338 in 2009, 12539 in 2011, and 11259 in 2015.

\section{Measurements}

Weight, height, WC and BP were measured following standardized protocols from the World Health Organization (WHO) ${ }^{17,18}$. WC was initially collected in 1993. Weight was measured with the participants wearing light clothing on a calibrated beam scale and height was measured without shoes using a portable stadiometer. BMI was calculated as weight in kilograms divided by height in meters squared. WC was measured with an inelastic tape to the nearest $0.1 \mathrm{~cm}$ at a midpoint between the bottom of the rib cage and the top of the iliac crest at the end of exhalation. BP was measured by trained technicians in triplicate after a 10-min rest, using mercury manometers. The three readings were averaged as the BP values in our data analysis. All physical examinations were performed at the same location and followed the same protocol at each study visit.

\section{Definitions}

According to $\mathrm{WHO}$ suggestions ${ }^{18}$, normal weight is defined as $\mathrm{BMI}<25 \mathrm{~kg} / \mathrm{m}^{2}$, general obesity is defined as $\mathrm{BMI} \geq 30 \mathrm{~kg} / \mathrm{m}^{2}$. According to WHO expert consultation for Asians ${ }^{19}$, normal weight is defined as $\mathrm{BMI}<23 \mathrm{~kg} / \mathrm{m}^{2}$. According to the criteria recommended by Working Group on Obesity in China ${ }^{20}$, general obesity is defined as $\mathrm{BMI} \geq 28 \mathrm{~kg} / \mathrm{m}^{2}$. According to the International Diabetes Federation recommendations for Asians ${ }^{21}$, central obesity is defined as WC $\geq 90 \mathrm{~cm}$ for men and $\geq 80 \mathrm{~cm}$ for women.

\section{Statistical analysis}

All statistical analyses were performed with SAS version 9.2 (SAS Institute Inc., Cary, North Carolina). Continuous variables were presented as means and standard deviations (SD). Categorical variables were expressed as numbers or percentages. One-way ANOVA was applied to compare differences in means across groups. A Chi-square test was performed to assess differences of proportions across groups. To take into account the changes in population age structure during the survey period, the estimated mean WC and prevalence of abdominal obesity were age-standardized to the 2000 census of the Chinese adult population by the direct method. Time trends for the mean WC across the surveys were assessed using linear regression models, with the year of the survey entered as a continuous variable. Trends in the prevalence of central obesity among participants with $\mathrm{BMI}<25 \mathrm{~kg} / \mathrm{m}^{2}$ from 1993 to 2015 were assessed by Cochran-Armitage trend testing. A sensitivity analysis was performed using a more stringent $\mathrm{BMI}<23 \mathrm{~kg} / \mathrm{m}^{2}$ cut point (Asian cut point). To assess whether changes throughout the 23-year period differed by sex, logistic regression analysis was utilized to examine potential interaction effects between cohort and sex. Similar processes were repeated separately for age groups, rural/urban regions, and educational attainment groups. Venn diagram was constructed as a visual display of how abdominal obesity defined by WC and general obesity defined by BMI clustered together. A two-tailed $P$ value of $<0.05$ was considered to be statistically significant.

\section{Results}

The percentage of women and the mean age of the study population increased progressively from 1993 to 2015 (all P<0.01) (Supplementary Table 1).

Trends in the age-standardized mean WC from 1993 to 2015 among Chinese adults with $\mathrm{BMI}<25 \mathrm{~kg} / \mathrm{m}^{2}$ were shown for overall and by sex, age, region categories, and educational attainment in Table 1. Overall, age-standardized mean WC increased by $3.4 \mathrm{~cm}$, (P for trend $<0.001)$. The increase in the age-standardized mean WC in men was greater than that in women $(P<0.001$ for interaction terms survey $\times$ sex $)$, with the agestandardized mean WC in men increased from $75.0 \mathrm{~cm}$ in 1993 to $81.2 \mathrm{~cm}$ in 2015, and in women from $72.8 \mathrm{~cm}$ in 1993 to $76.3 \mathrm{~cm}$ in 2015 . For each survey, the age-specific mean WC increased with age (all P for trend $<0.01$ ). Further, for each age group, the age-standardized mean WC increased linearly from 1993 to 2015 ( $P<0.001)$. Mean WC increased more in participants aged $\geq 65$ years than that in those aged $18-44$ years $(P$ $=0.043$ for interaction terms survey $\times$ age). The age-standardized mean WC increased over time in both rural and urban regions and in all educational attainment categories (all $\mathrm{P}$ for trends $<0.001)$. Notably, mean WC increased more in rural versus urban regions $(\mathrm{P}<0.001$ for interaction terms survey $\times$ rural/urban regions). 
Table 1

Trends in age-standardized mean waist circumference among Chinese adults with body mass index $<25 \mathrm{~kg} / \mathrm{m}^{2}, 1993-2015$.

\begin{tabular}{|c|c|c|c|c|c|c|c|c|c|c|}
\hline & 1993 & 1997 & 2000 & 2004 & 2006 & 2009 & 2011 & 2015 & $\begin{array}{l}P \text { for } \\
\text { trend }\end{array}$ & $\begin{array}{l}P \text { for } \\
\text { interaction }\end{array}$ \\
\hline Total & $\begin{array}{l}73.8 \pm \\
7.5\end{array}$ & $\begin{array}{l}74.8 \pm \\
7.5\end{array}$ & $\begin{array}{l}75.9 \pm \\
8.0\end{array}$ & $\begin{array}{l}76.6 \pm \\
8.1\end{array}$ & $\begin{array}{l}76.7 \pm \\
8.0\end{array}$ & $\begin{array}{l}77.4 \pm \\
8.3\end{array}$ & $\begin{array}{l}78.0 \pm \\
9.1\end{array}$ & $\begin{array}{l}78.2 \pm \\
11.1\end{array}$ & $<.0001$ & \\
\hline Men & $\begin{array}{l}75.0 \pm \\
7.2\end{array}$ & $\begin{array}{l}76.3 \pm \\
7.3\end{array}$ & $\begin{array}{l}77.6 \pm \\
7.8\end{array}$ & $\begin{array}{l}78.8 \pm \\
8.0\end{array}$ & $\begin{array}{l}78.9 \pm \\
7.8\end{array}$ & $\begin{array}{l}79.5 \pm \\
8.1\end{array}$ & $\begin{array}{l}80.6 \pm \\
9.0\end{array}$ & $\begin{array}{l}81.2 \pm \\
10.4\end{array}$ & $<.0001$ & $<.0001$ \\
\hline Women & $\begin{array}{l}72.8 \pm \\
7.6\end{array}$ & $\begin{array}{l}73.2 \pm \\
7.4\end{array}$ & $\begin{array}{l}74.2 \pm \\
7.9\end{array}$ & $\begin{array}{l}74.6 \pm \\
7.9\end{array}$ & $\begin{array}{l}74.8 \pm \\
7.9\end{array}$ & $\begin{array}{l}75.6 \pm \\
8.3\end{array}$ & $\begin{array}{l}76.1 \pm \\
8.8\end{array}$ & $\begin{array}{l}76.3 \pm \\
11.3\end{array}$ & $<.0001$ & \\
\hline \multicolumn{11}{|l|}{ Age (years) } \\
\hline $18-44$ & $\begin{array}{l}72.7 \pm \\
6.9\end{array}$ & $\begin{array}{l}74.0 \pm \\
7.0\end{array}$ & $\begin{array}{l}74.8 \pm \\
7.5\end{array}$ & $\begin{array}{l}75.9 \pm \\
7.8\end{array}$ & $\begin{array}{l}76.0 \pm \\
7.8\end{array}$ & $\begin{array}{l}76.4 \pm \\
8.1\end{array}$ & $\begin{array}{l}77.2 \pm \\
8.9\end{array}$ & $\begin{array}{l}77.5 \pm \\
10.9\end{array}$ & $<.0001$ & \multirow[t]{3}{*}{0.043} \\
\hline $45-64$ & $\begin{array}{l}75.7 \pm \\
7.6\end{array}$ & $\begin{array}{l}76.2 \pm \\
7.5\end{array}$ & $\begin{array}{l}78.0 \pm \\
7.7\end{array}$ & $\begin{array}{l}78.8 \pm \\
7.7\end{array}$ & $\begin{array}{l}79.0 \pm \\
7.5\end{array}$ & $\begin{array}{l}80.1 \pm \\
7.9\end{array}$ & $\begin{array}{l}80.5 \pm \\
9.0\end{array}$ & $\begin{array}{l}80.9 \pm \\
10.5\end{array}$ & $<.0001$ & \\
\hline $65-118$ & $\begin{array}{l}76.1 \pm \\
9.0\end{array}$ & $\begin{array}{l}76.5 \pm \\
8.9\end{array}$ & $\begin{array}{l}78.6 \pm \\
9.3\end{array}$ & $\begin{array}{l}78.5 \pm \\
9.1\end{array}$ & $\begin{array}{l}79.3 \pm \\
8.8\end{array}$ & $\begin{array}{l}80.4 \pm \\
8.7\end{array}$ & $\begin{array}{l}80.7 \pm \\
9.2\end{array}$ & $\begin{array}{l}80.7 \pm \\
11.7\end{array}$ & $<.0001$ & \\
\hline \multicolumn{11}{|l|}{ Region } \\
\hline Urban & $\begin{array}{l}74.8 \pm \\
8.4\end{array}$ & $\begin{array}{l}74.9 \pm \\
7.9\end{array}$ & $\begin{array}{l}75.9 \pm \\
8.6\end{array}$ & $\begin{array}{l}76.8 \pm \\
8.5\end{array}$ & $\begin{array}{l}77.1 \pm \\
8.3\end{array}$ & $\begin{array}{l}77.3 \pm \\
8.4\end{array}$ & $\begin{array}{l}78.1 \pm \\
9.2\end{array}$ & $\begin{array}{l}78.3 \pm \\
12.1\end{array}$ & $<.0001$ & \multirow[t]{2}{*}{$<.0001$} \\
\hline Rural & $\begin{array}{l}73.2 \pm \\
7.0\end{array}$ & $\begin{array}{l}74.5 \pm \\
7.3\end{array}$ & $\begin{array}{l}75.6 \pm \\
7.7\end{array}$ & $\begin{array}{l}76.3 \pm \\
7.9\end{array}$ & $\begin{array}{l}76.3 \pm \\
7.9\end{array}$ & $\begin{array}{l}77.1 \pm \\
8.3\end{array}$ & $\begin{array}{l}77.7 \pm \\
9.1\end{array}$ & $\begin{array}{l}77.8 \pm \\
10.4\end{array}$ & $<.0001$ & \\
\hline \multicolumn{11}{|l|}{ Education } \\
\hline $\begin{array}{l}\text { Less than high } \\
\text { school }\end{array}$ & $\begin{array}{l}73.8 \pm \\
7.5\end{array}$ & $\begin{array}{l}74.7 \pm \\
7.6\end{array}$ & $\begin{array}{l}75.7 \pm \\
7.9\end{array}$ & $\begin{array}{l}76.1 \pm \\
8.0\end{array}$ & $\begin{array}{l}76.2 \pm \\
8.0\end{array}$ & $\begin{array}{l}77.2 \pm \\
8.4\end{array}$ & $\begin{array}{l}77.5 \pm \\
9.3\end{array}$ & $\begin{array}{l}78.2 \pm \\
10.8\end{array}$ & $<.0001$ & \multirow[t]{3}{*}{0.51} \\
\hline High school & $\begin{array}{l}73.7 \pm \\
7.1\end{array}$ & $\begin{array}{l}74.7 \pm \\
7.2\end{array}$ & $\begin{array}{l}75.6 \pm \\
7.6\end{array}$ & $\begin{array}{l}76.3 \pm \\
8.0\end{array}$ & $\begin{array}{l}76.3 \pm \\
7.7\end{array}$ & $\begin{array}{l}76.7 \pm \\
8.1\end{array}$ & $\begin{array}{l}77.2 \pm \\
9.0\end{array}$ & $\begin{array}{l}77.6 \pm \\
10.7\end{array}$ & $<.0001$ & \\
\hline University & $\begin{array}{l}74.2 \pm \\
8.0\end{array}$ & $\begin{array}{l}75.2 \pm \\
7.8\end{array}$ & $\begin{array}{l}75.9 \pm \\
8.6\end{array}$ & $\begin{array}{l}76.6 \pm \\
8.4\end{array}$ & $\begin{array}{l}76.5 \pm \\
8.4\end{array}$ & $\begin{array}{l}76.8 \pm \\
8.5\end{array}$ & $\begin{array}{l}77.6 \pm \\
9.0\end{array}$ & $\begin{array}{l}77.8 \pm \\
11.7\end{array}$ & $<.0001$ & \\
\hline
\end{tabular}

Although with relatively lower WC values (Table 2), the trends in WC from 1993 to 2015 were similar among Chinese adults with BMI < $23 \mathrm{~kg} / \mathrm{m}^{2}$ (Asian cut point for normal weight). 
Table 2

Trends in age-standardized mean waist circumference among Chinese adults with body mass index $<23 \mathrm{~kg} / \mathrm{m}^{2}, 1993-2015$.

\begin{tabular}{|c|c|c|c|c|c|c|c|c|c|c|}
\hline & 1993 & 1997 & 2000 & 2004 & 2006 & 2009 & 2011 & 2015 & $\begin{array}{l}P \text { for } \\
\text { trend }\end{array}$ & $\begin{array}{l}P \text { for } \\
\text { interaction }\end{array}$ \\
\hline Total & $\begin{array}{l}72.5 \pm \\
6.8\end{array}$ & $\begin{array}{l}73.3 \pm \\
6.9\end{array}$ & $\begin{array}{l}74.1 \pm \\
7.2\end{array}$ & $\begin{array}{l}74.7 \pm \\
7.6\end{array}$ & $\begin{array}{l}74.8 \pm \\
7.4\end{array}$ & $\begin{array}{l}75.3 \pm \\
7.7\end{array}$ & $\begin{array}{l}75.7 \pm \\
8.5\end{array}$ & $\begin{array}{l}75.8 \pm \\
10.7\end{array}$ & $<.0001$ & \\
\hline Men & $\begin{array}{l}73.7 \pm \\
6.5\end{array}$ & $\begin{array}{l}74.9 \pm \\
6.6\end{array}$ & $\begin{array}{l}75.8 \pm \\
7.0\end{array}$ & $\begin{array}{l}76.6 \pm \\
7.4\end{array}$ & $\begin{array}{l}76.9 \pm \\
7.2\end{array}$ & $\begin{array}{l}77.1 \pm \\
7.5\end{array}$ & $\begin{array}{l}78.0 \pm \\
8.6\end{array}$ & $\begin{array}{l}78.7 \pm \\
10.1\end{array}$ & $<.0001$ & $<.0001$ \\
\hline Women & $\begin{array}{l}71.3 \pm \\
6.8\end{array}$ & $\begin{array}{l}71.8 \pm \\
6.8\end{array}$ & $\begin{array}{l}72.6 \pm \\
7.2\end{array}$ & $\begin{array}{l}73.0 \pm \\
7.4\end{array}$ & $\begin{array}{l}73.0 \pm \\
7.2\end{array}$ & $\begin{array}{l}73.8 \pm \\
7.7\end{array}$ & $\begin{array}{l}74.1 \pm \\
8.1\end{array}$ & $\begin{array}{l}74.2 \pm \\
10.8\end{array}$ & $<.0001$ & \\
\hline \multicolumn{11}{|l|}{ Age (years) } \\
\hline $18-44$ & $\begin{array}{l}71.5 \pm \\
6.3\end{array}$ & $\begin{array}{l}72.8 \pm \\
6.5\end{array}$ & $\begin{array}{l}73.3 \pm \\
6.9\end{array}$ & $\begin{array}{l}74.0 \pm \\
7.3\end{array}$ & $\begin{array}{l}74.2 \pm \\
7.3\end{array}$ & $\begin{array}{l}74.3 \pm \\
7.3\end{array}$ & $\begin{array}{l}74.9 \pm \\
8.2\end{array}$ & $\begin{array}{l}74.8 \pm \\
10.4\end{array}$ & $<.0001$ & \multirow[t]{3}{*}{0.0163} \\
\hline $45-64$ & $\begin{array}{l}74.1 \pm \\
6.9\end{array}$ & $\begin{array}{l}74.3 \pm \\
6.8\end{array}$ & $\begin{array}{l}75.7 \pm \\
7.0\end{array}$ & $\begin{array}{l}76.5 \pm \\
7.3\end{array}$ & $\begin{array}{l}76.5 \pm \\
6.8\end{array}$ & $\begin{array}{l}77.5 \pm \\
7.4\end{array}$ & $\begin{array}{l}77.9 \pm \\
8.4\end{array}$ & $\begin{array}{l}77.9 \pm \\
10.3\end{array}$ & $<.0001$ & \\
\hline $65-118$ & $\begin{array}{l}74.3 \pm \\
8.1\end{array}$ & $\begin{array}{l}74.7 \pm \\
8.1\end{array}$ & $\begin{array}{l}76.2 \pm \\
8.4\end{array}$ & $\begin{array}{l}76.2 \pm \\
8.4\end{array}$ & $\begin{array}{l}76.9 \pm \\
8.1\end{array}$ & $\begin{array}{l}78.1 \pm \\
8.1\end{array}$ & $\begin{array}{l}78.1 \pm \\
8.8\end{array}$ & $\begin{array}{l}78.2 \pm \\
11.1\end{array}$ & $<.0001$ & \\
\hline \multicolumn{11}{|l|}{ Region } \\
\hline Urban & $\begin{array}{l}73.2 \pm \\
7.8\end{array}$ & $\begin{array}{l}73.4 \pm \\
7.3\end{array}$ & $\begin{array}{l}74.2 \pm \\
7.9\end{array}$ & $\begin{array}{l}74.8 \pm \\
8.0\end{array}$ & $\begin{array}{l}75.0 \pm \\
7.7\end{array}$ & $\begin{array}{l}75.2 \pm \\
7.8\end{array}$ & $\begin{array}{l}75.9 \pm \\
8.9\end{array}$ & $\begin{array}{l}75.9 \pm \\
11.1\end{array}$ & $<.0001$ & \multirow[t]{2}{*}{0.2655} \\
\hline Rural & $\begin{array}{l}72.0 \pm \\
6.3\end{array}$ & $\begin{array}{l}73.2 \pm \\
6.6\end{array}$ & $\begin{array}{l}73.9 \pm \\
6.9\end{array}$ & $\begin{array}{l}74.6 \pm \\
7.4\end{array}$ & $\begin{array}{l}74.5 \pm \\
7.2\end{array}$ & $\begin{array}{l}75.1 \pm \\
7.6\end{array}$ & $\begin{array}{l}75.3 \pm \\
8.3\end{array}$ & $\begin{array}{l}75.3 \pm \\
10.4\end{array}$ & $<.0001$ & \\
\hline \multicolumn{11}{|l|}{ Education } \\
\hline $\begin{array}{l}\text { Less than high } \\
\text { school }\end{array}$ & $\begin{array}{l}72.4 \pm \\
6.7\end{array}$ & $\begin{array}{l}73.3 \pm \\
6.9\end{array}$ & $\begin{array}{l}74.1 \pm \\
7.2\end{array}$ & $\begin{array}{l}74.4 \pm \\
7.4\end{array}$ & $\begin{array}{l}74.4 \pm \\
7.3\end{array}$ & $\begin{array}{l}75.2 \pm \\
7.7\end{array}$ & $\begin{array}{l}75.4 \pm \\
8.7\end{array}$ & $\begin{array}{l}75.9 \pm \\
10.6\end{array}$ & $<.0001$ & \multirow[t]{3}{*}{0.63} \\
\hline High school & $\begin{array}{l}72.6 \pm \\
6.6\end{array}$ & $\begin{array}{l}73.5 \pm \\
6.7\end{array}$ & $\begin{array}{l}73.9 \pm \\
6.8\end{array}$ & $\begin{array}{l}74.6 \pm \\
7.6\end{array}$ & $\begin{array}{l}74.6 \pm \\
7.0\end{array}$ & $\begin{array}{l}74.7 \pm \\
7.5\end{array}$ & $\begin{array}{l}75.1 \pm \\
8.5\end{array}$ & $\begin{array}{l}75.2 \pm \\
10.5\end{array}$ & $<.0001$ & \\
\hline University & $\begin{array}{l}72.8 \pm \\
7.3\end{array}$ & $\begin{array}{l}73.3 \pm \\
6.9\end{array}$ & $\begin{array}{l}74.3 \pm \\
8.1\end{array}$ & $\begin{array}{l}74.6 \pm \\
8.0\end{array}$ & $\begin{array}{l}74.5 \pm \\
7.8\end{array}$ & $\begin{array}{l}74.8 \pm \\
7.8\end{array}$ & $\begin{array}{l}75.3 \pm \\
8.4\end{array}$ & $\begin{array}{l}75.1 \pm \\
10.9\end{array}$ & $<.0001$ & \\
\hline
\end{tabular}

In 1993, the age-standardized prevalence of abdominal obesity among Chinese adults with $\mathrm{BMI}<25 \mathrm{~kg} / \mathrm{m}^{2}$ was $11.2 \%$. By 2015 , the prevalence was more than doubled. Prevalence of abdominal obesity increased by a larger amount in men, from $3.7 \%$ in 1993 to $12.9 \%$ in 2015 in men, and from $18.6 \%$ in 1993 to $33.6 \%$ in 2015 in women (Fig. 1). The prevalence of abdominal obesity increased significantly between 1993 and 2015 in all studied subgroups (all P for trends < 0.001) (Fig. 1). Of note, trends in the prevalence of abdominal obesity from 1993 to 2015 increased more in younger participants than that in those aged $\geq 65$ years $(P<0.001$ for interaction terms survey $\times$ age $)$, and in rural than that in urban regions $(P<$ 0.001 for interaction terms survey $\times$ rural/urban regions).

The prevalence of abdominal obesity also worsened overtime when a more stringent $\mathrm{BMI}<23 \mathrm{~kg} / \mathrm{m}^{2}$ cut point was applied to define normal weight (Fig. 2).

How abdominal obesity defined by $\mathrm{WC} \geq 90 \mathrm{~cm}$ in men and $80 \mathrm{~cm}$ in women and general obesity defined by BMI $\geq 28 \mathrm{~kg} / \mathrm{m}^{2} \mathrm{clustered} \mathrm{together} \mathrm{for}$ each survey was showed in Table 3. For each survey, among those subjects with either abdominal obesity or general obesity, considerable participants (more than 66\%) had exclusive abdominal obesity compared with very rare (less than $2 \%$ ) with exclusive general obesity. A low magnitude of overlap (less than 26\%) existed between abdominal obesity and general obesity. Similar phenomena of how abdominal obesity and general obesity clustered together were noted in both genders for each survey. Exclusive general obesity was even less frequent in men than in women. 
Table 3

Overlap between body mass index- and waist circumference-based obesity among Chinese adults [

\begin{tabular}{|c|c|c|c|c|c|c|c|c|c|c|c|c|c|c|c|c|}
\hline & \multicolumn{2}{|l|}{1993} & \multicolumn{2}{|l|}{1997} & \multicolumn{2}{|l|}{2000} & \multicolumn{2}{|l|}{2004} & \multicolumn{2}{|l|}{2006} & \multicolumn{2}{|l|}{2009} & \multicolumn{2}{|l|}{2011} & \multicolumn{2}{|l|}{2015} \\
\hline & $n$ & $\%$ & $\mathrm{n}$ & $\%$ & $n$ & $\%$ & $\mathrm{n}$ & $\%$ & $\mathrm{n}$ & $\%$ & $n$ & $\%$ & $n$ & $\%$ & $n$ & $\%$ \\
\hline \multicolumn{17}{|l|}{ Total } \\
\hline $\begin{array}{l}\mathrm{BMI} \geq \\
28 \mathrm{~kg} / \mathrm{m}^{2} \text { or } \\
W C \geq \\
90 / 80 \mathrm{~cm}\end{array}$ & 1513 & $\ldots$ & 2035 & $\ldots$ & 2949 & $\ldots$ & 3241 & $\ldots$ & 3399 & $\ldots$ & 4033 & $\ldots$ & 6034 & $\ldots$ & 6023 & $\ldots$ \\
\hline $\begin{array}{l}\text { Exclusive } \\
\text { BMI } \geq \\
28 \mathrm{~kg} / \mathrm{m}^{2}\end{array}$ & 22 & 1.5 & 23 & 1.1 & 39 & 1.3 & 37 & 1.1 & 51 & 1.5 & 35 & 0.9 & 102 & 1.7 & 78 & 1.3 \\
\hline $\begin{array}{l}\text { Exclusive } \\
W C \geq \\
90 / 80 \mathrm{~cm}\end{array}$ & 1272 & 84.1 & 1610 & 79.1 & 2319 & 78.6 & 2527 & 78.0 & 2665 & 78.4 & 3145 & 78.0 & 4527 & 75.0 & 4392 & 72.9 \\
\hline $\begin{array}{l}\text { Both } \mathrm{BMI} \geq \\
28 \mathrm{~kg} / \mathrm{m}^{2} \text { and } \\
\mathrm{WC} \geq \\
90 / 80 \mathrm{~cm}\end{array}$ & 219 & 14.5 & 402 & 19.8 & 591 & 20.0 & 677 & 20.9 & 683 & 20.1 & 853 & 21.2 & 1405 & 23.3 & 1553 & 25.8 \\
\hline \multicolumn{17}{|l|}{ Men } \\
\hline $\begin{array}{l}\mathrm{BMI} \geq \\
28 \mathrm{~kg} / \mathrm{m}^{2} \text { or } \\
W C \geq \\
90 / 80 \mathrm{~cm}\end{array}$ & 343 & $\ldots$ & 612 & $\ldots$ & 948 & $\ldots$ & 1046 & $\ldots$ & 1102 & $\ldots$ & 1372 & $\ldots$ & 2207 & $\ldots$ & 2170 & $\ldots$ \\
\hline $\begin{array}{l}\text { Exclusive } \\
\text { BMI } \geq \\
28 \mathrm{~kg} / \mathrm{m}^{2}\end{array}$ & 13 & 3.8 & 17 & 2.8 & 28 & 3.0 & 27 & 2.6 & 39 & 3.5 & 28 & 2.0 & 68 & 3.1 & 62 & 2.9 \\
\hline $\begin{array}{l}\text { Exclusive } \\
\text { WC } \geq \\
90 / 80 \mathrm{~cm}\end{array}$ & 264 & 77.0 & 428 & 69.9 & 679 & 71.6 & 755 & 72.2 & 800 & 72.6 & 990 & 72.2 & 1536 & 69.6 & 1435 & 66.1 \\
\hline $\begin{array}{l}\text { Both } \mathrm{BMI} \geq \\
28 \mathrm{~kg} / \mathrm{m}^{2} \text { and } \\
\mathrm{WC} \geq \\
90 / 80 \mathrm{~cm}\end{array}$ & 66 & 19.2 & 167 & 27.3 & 241 & 25.4 & 264 & 25.2 & 263 & 23.9 & 354 & 25.8 & 603 & 27.3 & 673 & 31.0 \\
\hline \multicolumn{17}{|l|}{ Women } \\
\hline $\begin{array}{l}\mathrm{BMI} \geq \\
28 \mathrm{~kg} / \mathrm{m}^{2} \text { or } \\
W C \geq \\
90 / 80 \mathrm{~cm}\end{array}$ & 1170 & $\ldots$ & 1423 & $\ldots$ & 2001 & $\ldots$ & 2195 & $\ldots$ & 2297 & $\ldots$ & 2661 & $\ldots$ & 3827 & $\ldots$ & 3853 & $\ldots$ \\
\hline $\begin{array}{l}\text { Exclusive } \\
\text { BMI } \geq \\
28 \mathrm{~kg} / \mathrm{m}^{2}\end{array}$ & 9 & 0.8 & 6 & 0.4 & 11 & 0.5 & 10 & 0.5 & 12 & 0.5 & 7 & 0.3 & 34 & 0.9 & 16 & 0.4 \\
\hline $\begin{array}{l}\text { Exclusive } \\
\text { WC } \geq \\
90 / 80 \mathrm{~cm}\end{array}$ & 1008 & 86.2 & 1182 & 83.1 & 1640 & 82.0 & 1772 & 80.7 & 1865 & 81.2 & 2155 & 81.0 & 2991 & 78.2 & 2957 & 76.7 \\
\hline $\begin{array}{l}\text { Both } \mathrm{BMI} \geq \\
28 \mathrm{~kg} / \mathrm{m}^{2} \\
\text { and } \mathrm{WC} \geq \\
90 / 80 \mathrm{~cm}\end{array}$ & 153 & 13.1 & 235 & 16.5 & 350 & 17.5 & 413 & 18.8 & 420 & 18.3 & 499 & 18.8 & 802 & 21.0 & 880 & 22.8 \\
\hline $\begin{array}{l}\text { Proportions o } \\
\text { number of obe } \\
\geq 28 \mathrm{~kg} / \mathrm{m}^{2} \text {. }\end{array}$ & $\begin{array}{l}\text { besity } \\
\text { y base }\end{array}$ & $\begin{array}{l}\text { ased } \\
\text { on bc }\end{array}$ & $\begin{array}{l}n \text { both } \\
W C \geq\end{array}$ & $\begin{array}{l}f \geq 90 \\
/ 80\end{array}$ & $\begin{array}{l}80 \mathrm{~cm} \\
\text { and } \mathrm{B}\end{array}$ & $\begin{array}{l}d B M \\
I \geq 28\end{array}$ & $\begin{array}{l}\geq 28 \mathrm{~kg} \\
\mathrm{~g} / \mathrm{m}^{2}\end{array}$ & $\begin{array}{l}n^{2} \text { (ov } \\
\text { ided }\end{array}$ & $\begin{array}{l}\text { lap be } \\
\text { the nu }\end{array}$ & $\begin{array}{l}\text { een } B / \\
\text { ber of }\end{array}$ & $\begin{array}{l}\text { I- and V } \\
\text { besity }\end{array}$ & $\begin{array}{l}\text { C-base } \\
\text { ased o }\end{array}$ & $\begin{array}{l}\text { obesit } \\
\text { either }\end{array}$ & $\begin{array}{l}\text { is cal } \\
C \geq 90\end{array}$ & $\begin{array}{l}\text { lated a } \\
80 \mathrm{~cm}\end{array}$ & $\begin{array}{l}\text { the } \\
\text { BMI }\end{array}$ \\
\hline
\end{tabular}

Results were remarkably similar when using the $\mathrm{BMI} \geq 30 \mathrm{~kg} / \mathrm{m}^{2}$ cut point rather than the more restrictive Asian $\mathrm{BMI} \geq 28 \mathrm{~kg} / \mathrm{m}^{2} \mathrm{cut}$ point to define general obesity (data not shown). 
The present study, in which the most recent data from CHNS, including the 2015 survey, were analyzed, showed that mean WC, and the prevalence of abdominal obesity have increased dramatically among Chinese adults with normal weight, irrespective of which criterion was used, since 1993. The increases occurred in both genders, all age groups, rural and urban residents, and all educational attainment groups. Moreover, men, younger participants, and rural residents showed relatively greater increases. Our results are of particular concern as abdominal obesity, which reasonably represents visceral adiposity, is closely associated with obesity-related conditions and mortality in those with a normal $\mathrm{BMI}^{8}$.

Although few studies have analyzed trends in mean WC and prevalence of abdominal obesity among people with acceptable BMI, our results are generally in line with those of previous studies showing that a considerable proportion of individuals suffered from abdominal obesity among people with normal $\mathrm{BMI}^{22}$. Further, emerging evidence showed that WC increased much faster than BMI during the same periods, and hence the relative increase in abdominal obesity at the same periods is much larger than that of general obesity ${ }^{23,24}$. Our previous study showed a dramatic upward trend in the prevalence of abdominal obesity among people with normal BMI from 1993 to $2009^{14}$. The present study showed that the increasing trend from 2009 to 2015 appeared to continue rather than slow or level off. Our results together with previous reports suggest that body composition has changed over time. Evidence showed that approximately $20 \%$ higher risk of mortality occurred in individuals with normal BMI who were abdominally obese compared with their counterparts with normal BMI who were not abdominally obese ${ }^{25}$ and that the association between WC and mortality was strongest in those with a normal $\mathrm{BMI}^{8}$. Thus, depicting the changing trend of abdominal obesity among people with acceptable BMI may provide additional information for more accurately assessing the prevalence of obesity-related disorders. Considering the more deleterious effect of visceral fat on metabolic disorders than subcutaneous fat ${ }^{26-29}$, the increase in WC is likely to be due to a relatively greater increase in visceral adipose tissue than that of subcutaneous fat. Therefore, it is urgent to take interventional strategies to reverse abdominal obesity trends and reduce the likely medical costs of the increase in abdominal obesity in normal-weight people.

The continuing rapid increase in the prevalence of abdominal obesity among individuals with normal BMI in China from 1993 to 2015 is attributed to several factors. Unhealthy lifestyles and behavioral changes are probably major drivers. Increased availability, accessibility, and affordability of energy-dense foods and a more sedentary lifestyle that have followed urbanization and increasingly mechanized transportation and labor are responsible for excess energy intake and reduced energy expenditure, respectively, and thus induce fat accumulation in the body. For example, China has been experiencing westernization of their diet. As a result, the consumption of plant-based foods such as cereals and starchy roots has dramatically declined; in contrast, the intake of foods rich in sugar, fat, and refined carbohydrates as well as animal-based food such as red meat, and processed meat has dramatically increased ${ }^{30}$. Active transportation, such as walking or cycling, which was associated with a decrease in obesity and weight gain, covered up to $80 \%$ of daily travel in China until the 1990 s, but this situation declined dramatically thereafter 31,32 . TV ownership increased dramatically during the recent two decades, with 38 sets per 1,000 persons in 1985 and 112 to 135 sets per 100 households in $2011^{33}$. Future unfavorable trends in dietary pattern and physical activity level will exacerbate the increase in the prevalence of abdominal obesity.

Our findings that the rates of such an increase in abdominal obesity prevalence varied by sex, age, and rural/urban regions are also a characteristic noted in other studies ${ }^{1,23,34}$. Although the root causes that induce the difference is not clear, disparities between subgroups in genetic, sociocultural, socioeconomic, and behavioral factors, such as disparities in calorie intake, knowledge and means to adopt healthy lifestyles as well as weight management programs, and mechanized transport and work, have been considered as potential drivers ${ }^{30}$. Despite the inequalities in the abdominal obesity prevalence by sex, age, and rural/urban regions, our study showed that the abdominal obesity prevalence increased over time in all subgroups, indicating a leading role of the obesogenic environment in China in the recent two decades. Further, our finding that the continuous increase in the prevalence of abdominal obesity was much faster in younger people is concerning because it predicts the prevalence of abdominal obesity prevalence among normal-weight people should keep increasing in the next few years.

In the present study, a significant dissociation between general obesity defined by BMI and abdominal obesity defined by WC was noted, with approximately $25 \%$ with the presence of both general obesity and abdominal obesity occurring together. Further, approximately two-thirds of individuals with obesity would be missed if WC is not taken into account for the identification of obesity. Hence, the absence of a WC measurement might result in substantial misclassification of individuals who are actually in a risk category based on WC as being in a low-risk category based on an acceptable range of BMI. Indeed, our previous study together with other reports evidenced that more than $20 \%$ of the normal weight population encountered an increased WC and a cluster of cardiovascular risk factors, including insulin resistance, atherogenic lipid profiles, hypertension, and non-acholic fatty liver disease ${ }^{9}$. Since increased WC and the mentioned cardiovascular risk factors exhibited increased incidences of diabetes, cardiovascular diseases, and all-cause mortality ${ }^{35-38}$, adding WC measurement into the BMI assessment would contribute to assessing whether an individual was actually in a risk state and thus could provide an opportunity for proper intervention.

Our study has several strengths. First, it maintains a large sample size and includes individuals from diverse and representative regions in China, which allows for exploring the prevalence of obesity over a range of demographic groups. Second, all study measurements are made by trained staff following a standard protocol. A vigorous quality assurance program and the same sampling and strict methodology are used to ensure the quality of the data collection over the entire study period, allowing direct comparisons of results over time. Third, we assessed the secular trends in abdominal obesity in people with normal BMI, using different cut-off points for BMI and identified consistent associations. However, the limitations of the present study require careful consideration. Firstly, the sample is partial nationally representative, therefore the generalizability of the results 
to regions not studied may be limited. Secondly, other social and environmental variables such as dietary habits, sleep duration, and physical activity, which would have an impact on obesity, were not considered. Third, measurements of body fatness were not available.

\section{Conclusions}

Mean WC and the prevalence of abdominal obesity increased significantly among Chinese adults with normal BMI from 1993 to 2015 . The increasing trends were observed in both genders, all age groups, both rural and urban regions, and all educational attainment groups. Men, younger participants, and rural residents experienced greater increases.

\section{List Of Abbreviations}

BMI

body mass index, WC:waist circumference, CHNS:China Health and Nutrition Survey, BP:blood pressure, WHO:World Health Organization, SD:standard deviation

\section{Declarations}

\section{Competing interests}

The authors declare that they have no competing interests.

\section{Authors' contributions}

TT.D and XX.S conceived the study. XX.S completed all statistical analyses and drafted the manuscript. TT.D revised the manuscript. All authors have read and approved the final manuscript.

\section{Acknowledgments}

We thank the China Health and Nutrition Survey, supported by the NIH (R01-D30880, DK056350, and R01-HD38700), and the National Institute of Nutrition and Food Safety, China Center for Disease Control and Prevention, Carolina Population Center, the University of North Carolina at Chapel Hill and the Fogarty International Center for providing the data used here. We also thank the China-Japan Friendship Hospital and Ministry of Health for support for the CHNS 2009 survey. The study was supported by the National Natural Science Foundation of China 81700762. All errors are the authors' alone.

\section{References}

1. Ma, S. et al. Trends in the prevalence of overweight, obesity, and abdominal obesity among Chinese adults between 1993 and 2015. International journal of obesity, doi:10.1038/s41366-020-00698-x (2020).

2. Wu, Y. Overweight and obesity in China. Bmj 333, 362-363, doi:10.1136/bmj.333.7564.362 (2006).

3. NCD Risk Factor Collaboration. Trends in adult body-mass index in 200 countries from 1975 to 2014: a pooled analysis of 1698 populationbased measurement studies with 19.2 million participants. Lancet 387, 1377-1396, doi:10.1016/S0140-6736(16)30054-X (2016).

4. GBD 2015 Obesity Collaborators. et al.Health Effects of Overweight and Obesity in 195 Countries over 25 Years. The New England journal of medicine 377, 13-27, doi:10.1056/NEJMoa1614362 (2017).

5. Yusuf, S. et al. Obesity and the risk of myocardial infarction in 27,000 participants from 52 countries: a case-control study. Lancet $366,1640-$ 1649, doi:10.1016/S0140-6736(05)67663-5 (2005).

6. Li, G. et al. Obesity, coronary heart disease risk factors and diabetes in Chinese: an approach to the criteria of obesity in the Chinese population. Obesity reviews: an official journal of the International Association for the Study of Obesity 3, 167-172, doi:10.1046/j.1467-789x.2002.00067.x (2002).

7. Zhang, X. et al. General and abdominal adiposity and risk of stroke in Chinese women. Stroke 40, 1098-1104, doi:10.1161/STROKEAHA.108.539692 (2009).

8. Jacobs, E. J. et al. Waist circumference and all-cause mortality in a large US cohort. Archives of internal medicine 170, 1293-1301, doi:10.1001/archinternmed.2010.201 (2010).

9. Wildman, R. P. et al. The obese without cardiometabolic risk factor clustering and the normal weight with cardiometabolic risk factor clustering: prevalence and correlates of 2 phenotypes among the US population (NHANES 1999-2004). Archives of internal medicine 168, 1617-1624, doi:10.1001/archinte.168.15.1617 (2008).

10. Kuk, J. L. \& Ardern, C. I. Are metabolically normal but obese individuals at lower risk for all-cause mortality? Diabetes care 32, 2297-2299, doi:10.2337/dc09-0574 (2009). 
11. Hinnouho, G. M., Czernichow, S. \& Singh-Manoux, A. Response to comment on Hinnouho et al. Metabolically healthy obesity and risk of mortality: does the definition of metabolic health matter? Diabetes care 2013;36:2294-2300. Diabetes care 37, e105, doi:10.2337/dc14-0101 (2014).

12. Yang, W. et al. Prevalence of diabetes among men and women in China. The New England journal of medicine 362, 1090-1101, doi:10.1056/NEJMoa0908292 (2010).

13. Xi, B. et al. Secular trends in the prevalence of general and abdominal obesity among Chinese adults, 1993-2009. Obesity reviews: an official journal of the International Association for the Study of Obesity 13, 287-296, doi:10.1111/j.1467-789X.2011.00944.x (2012).

14. Du, T. et al. Increasing trends in central obesity among Chinese adults with normal body mass index, 1993-2009. BMC public health 13, 327, doi:10.1186/1471-2458-13-327 (2013).

15. Nazare, J. A. et al. Ethnic influences on the relations between abdominal subcutaneous and visceral adiposity, liver fat, and cardiometabolic risk profile: the International Study of Prediction of Intra-Abdominal Adiposity and Its Relationship With Cardiometabolic Risk/Intra-Abdominal Adiposity. The American journal of clinical nutrition 96, 714-726, doi:10.3945/ajcn.112.035758 (2012).

16. Popkin, B. M., Du, S., Zhai, F. \& Zhang, B. Cohort Profile: The China Health and Nutrition Survey-monitoring and understanding socio-economic and health change in China, 1989-2011. International journal of epidemiology 39, 1435-1440, doi:10.1093/ije/dyp322 (2010).

17. Chobanian, A. V. et al. The Seventh Report of the Joint National Committee on Prevention, Detection, Evaluation, and Treatment of High Blood Pressure: the JNC 7 report. Jama 289, 2560-2572, doi:10.1001/jama.289.19.2560 (2003).

18. Physical status: the use and interpretation of anthropometry. Report of a WHO Expert Committee. World Health Organization technical report series $854,1-452(1995)$.

19. WHO Expert Consultation. Appropriate body-mass index for Asian populations and its implications for policy and intervention strategies. Lancet 363, 157-163, doi:10.1016/S0140-6736(03)15268-3 (2004).

20. Zhou, B. F. \& Cooperative Meta-Analysis Group of the Working Group on Obesity in, C. Predictive values of body mass index and waist circumference for risk factors of certain related diseases in Chinese adults-study on optimal cut-off points of body mass index and waist circumference in Chinese adults. Biomedical and environmental sciences: BES 15, 83-96 (2002).

21. Alberti, K. G., Zimmet, P., Shaw, J. \& Group, I. D. F. E. T. F. C. The metabolic syndrome-a new worldwide definition. Lancet 366, 1059-1062, doi:10.1016/S0140-6736(05)67402-8 (2005).

22. Booth, M. L., Hunter, C., Gore, C. J., Bauman, A. \& Owen, N. The relationship between body mass index and waist circumference: implications for estimates of the population prevalence of overweight. International journal of obesity and related metabolic disorders: journal of the International Association for the Study of Obesity 24, 1058-1061, doi:10.1038/sj.ijo.0801359 (2000).

23. Gutierrez-Fisac, J. L. et al. Prevalence of general and abdominal obesity in the adult population of Spain, 2008-2010: the ENRICA study. Obesity reviews: an official journal of the International Association for the Study of Obesity 13, 388-392, doi:10.1111/j.1467789X.2011.00964.x (2012).

24. Walls, H. L. et al. Comparing trends in BMI and waist circumference. Obesity 19, 216-219, doi:10.1038/oby.2010.149 (2011).

25. Koster, A. et al. Waist circumference and mortality. American journal of epidemiology 167, 1465-1475, doi:10.1093/aje/kwn079 (2008).

26. Smith, J. D. et al. Visceral adipose tissue indicates the severity of cardiometabolic risk in patients with and without type 2 diabetes: results from the INSPIRE ME IAA study. The Journal of clinical endocrinology and metabolism 97, 1517-1525, doi:10.1210/jc.2011-2550 (2012).

27. Liu, J. et al. Impact of abdominal visceral and subcutaneous adipose tissue on cardiometabolic risk factors: the Jackson Heart Study. The Journal of clinical endocrinology and metabolism 95, 5419-5426, doi:10.1210/jc.2010-1378 (2010).

28. Fox, C. S. et al. Abdominal visceral and subcutaneous adipose tissue compartments: association with metabolic risk factors in the Framingham Heart Study. Circulation 116, 39-48, doi:10.1161/CIRCULATIONAHA.106.675355 (2007).

29. Boyko, E. J., Fujimoto, W. Y., Leonetti, D. L. \& Newell-Morris, L. Visceral adiposity and risk of type 2 diabetes: a prospective study among Japanese Americans. Diabetes care 23, 465-471, doi:10.2337/diacare.23.4.465 (2000).

30. Li, Y. et al. Potential Impact of Time Trend of Life-Style Factors on Cardiovascular Disease Burden in China. Journal of the American College of Cardiology 68, 818-833, doi:10.1016/j.jacc.2016.06.011 (2016).

31. Ng, S. W., Norton, E. C. \& Popkin, B. M. Why have physical activity levels declined among Chinese adults? Findings from the $1991-2006$ China Health and Nutrition Surveys. Social science \& medicine 68, 1305-1314, doi:10.1016/j.socscimed.2009.01.035 (2009).

32. Smith, R. Creative destruction: capitalist development and China's environment. New Left Review, 3-42 (1997).

33. National Bureau of Statistics of China.China statistical yearbook, <http://www.stats.gov.cn/tjsj/ndsj/> (1996-2012).

34. Reynolds, K. et al. Prevalence and risk factors of overweight and obesity in China. Obesity 15, 10-18, doi:10.1038/oby.2007.527 (2007).

35. Hamer, M. \& Stamatakis, E. Metabolically healthy obesity and risk of all-cause and cardiovascular disease mortality. The Journal of clinical endocrinology and metabolism 97, 2482-2488, doi:10.1210/jc.2011-3475 (2012).

36. Panagiotakos, D. B., Pitsavos, C., Yannakoulia, M., Chrysohoou, C. \& Stefanadis, C. The implication of obesity and central fat on markers of chronic inflammation: The ATTICA study. Atherosclerosis 183, 308-315, doi:10.1016/j.atherosclerosis.2005.03.010 (2005).

Page $9 / 11$ 
37. Farin, H. M., Abbasi, F. \& Reaven, G. M. Body mass index and waist circumference correlate to the same degree with insulin-mediated glucose uptake. Metabolism: clinical and experimental 54, 1323-1328, doi:10.1016/j.metabol.2005.04.021 (2005).

38. Wannamethee, S. G., Shaper, A. G., Morris, R. W. \& Whincup, P. H. Measures of adiposity in the identification of metabolic abnormalities in elderly men. The American journal of clinical nutrition 81, 1313-1321, doi:10.1093/ajcn/81.6.1313 (2005).

Figures
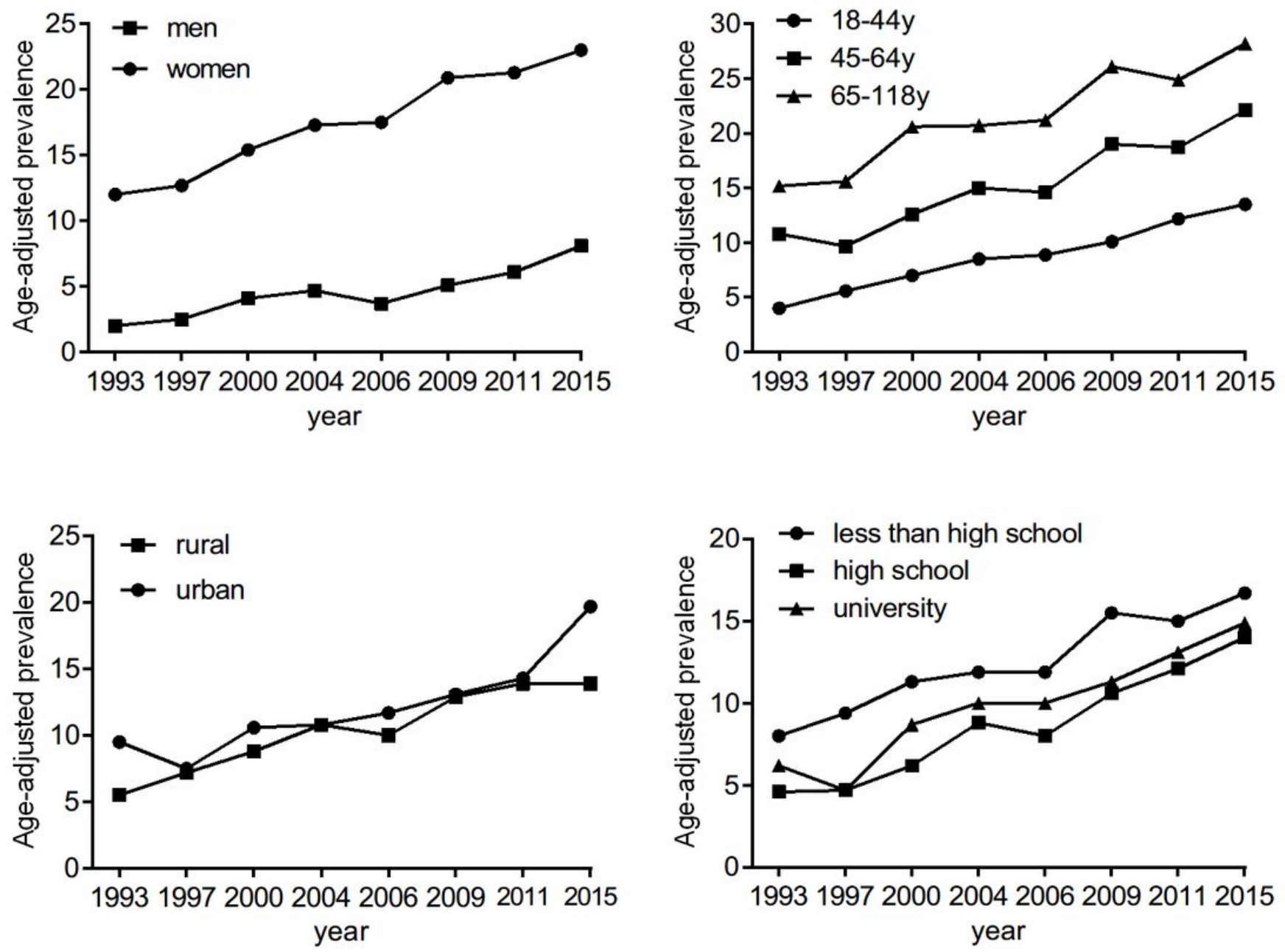

Figure 1

Trends in the age-adjusted prevalence of abdominal obesity among Chinese adults with body mass index $<25 \mathrm{~kg} / \mathrm{m} 2 \mathrm{by} \mathrm{sex}$, age, rural/urban setting, and educational attainment: the CHNS 1993-2015. 

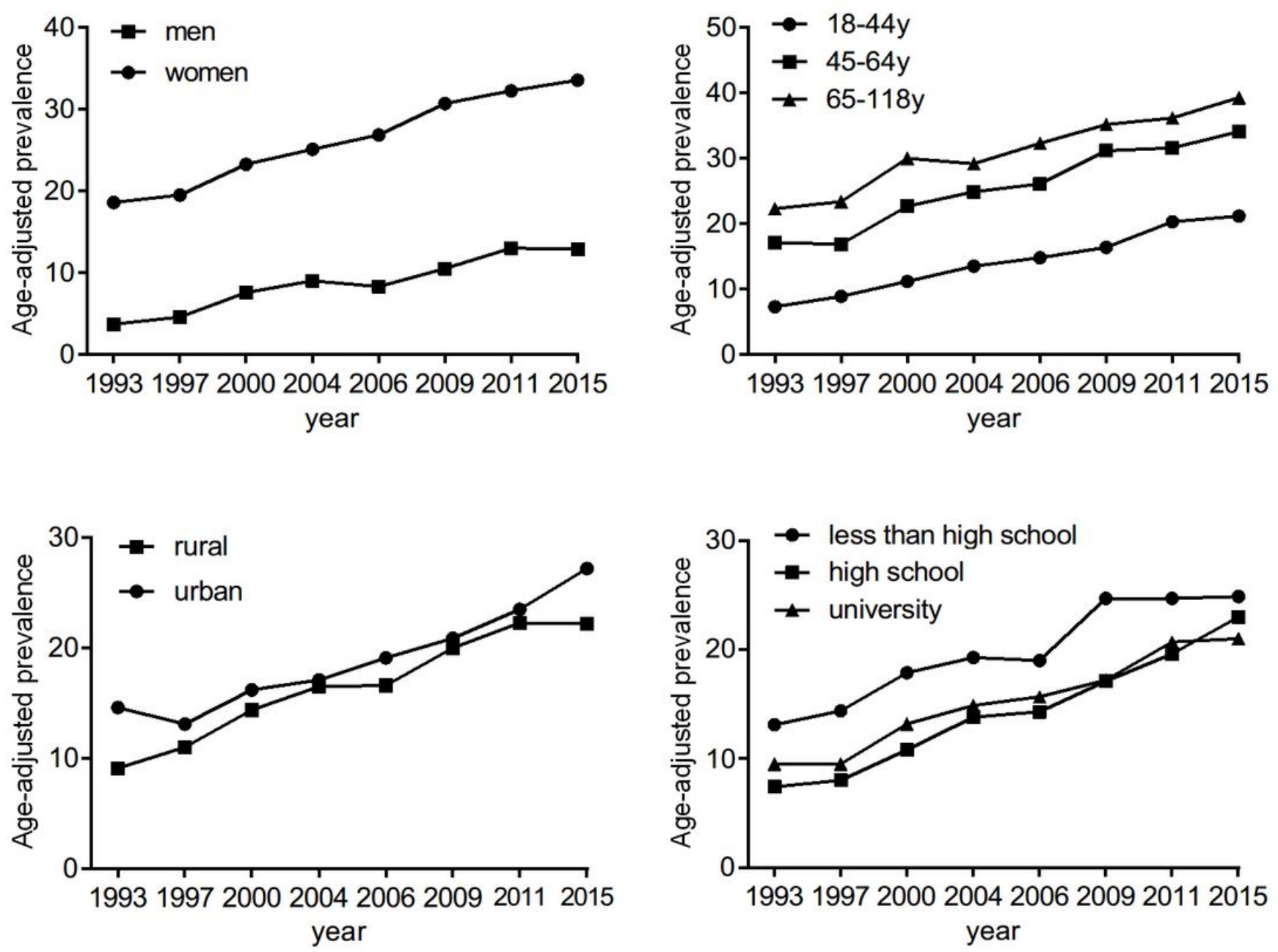

Figure 2

Trends in the age-adjusted prevalence of abdominal obesity among Chinese adults with body mass index $<23 \mathrm{~kg} / \mathrm{m} 2 \mathrm{by}$ sex, age, rural/urban setting, and educational attainment: the CHNS 1993-2015.

\section{Supplementary Files}

This is a list of supplementary files associated with this preprint. Click to download.

- SupplementaryTable1.docx 